\title{
GROUP RINGS WITH STRONGLY 2-GENERATED AUGMENTATION IDEALS
}

\author{
REFAAT M. SALEM
}

\begin{abstract}
Suppose that $G$ is a finite supersolvable (infinite solvable) group, $K$ is a field of char $p>0$. Then the Augmentation ideal $w(K[G])$ is right strongly 2 -generated iff $G$ is a $p^{\prime}$-group-by-cyclic $P$-group (finite $p^{\prime}$-group-by-infinite cyclic).
\end{abstract}

\section{Introduction}

A right ideal $I$ of a ring $R$ is called right strongly 2-generated if for any nonzero element $x \in \mathbb{I}$, there exists an element $y \in \mathbb{I}$, such that $\mathbb{I}=x \mathbb{R}+y \mathbb{R}$. This property is inherited by homomorphic images. Passman [3] has found that in the Noetherian group ring $\mathbb{K}[G]$ over any field $K$, the Augmentation ideal $w(\mathbb{K}[G])$ is right principal ideal iff $G$ is a finite $p^{\prime}$-group-by-cyclic group. Farkas and Snider [1] have answered the next question as to when the augmentation ideal was principal in the absence of the Noetherian condition.

In what follows we shall extend these results to the strongly 2-generated case. A group $G$ is called a $\pi$-group, where $\pi$ is a set of prime numbers, if $|G|$ is divisible by each prime of $\pi$. It is well known in the group ring $\mathbb{K}|G|$, over any field $K$, that right Noetherian implies left Noetherian, since it admits a ring antiautomorphism.

Proposition 1. Suppose that $G$ is a fnite supersolvable group of order $n p^{\tau}$, where $n>p, p \mid n$ and $K$ is a field of char $p>0$, then the following are

Received November 25, 1991. 
equivalent.

(i) $w(K[G])$ is a right strongly 2-generated ideal.

(ii) $G$ is a $p^{\prime}$-group-by-cyclic p-group.

(iii) $K[G]$ is a right principal ideal ring.

Proof.

(i)--(ii) Since $G$ is a finite supersolvable group it has a normal series, $G=$ $G_{0} \triangleright G_{1} \triangleright-\triangleright G_{n}=\langle 1\rangle$, with 1 the identity of $G$, such that $G_{i} / G_{i+1}$ is a cyclic group of prime order. Hence $N=G_{n-1}$ is a cyclic normal subgroup of prime order. The group epimorphism $\beta: G \rightarrow G / N$ can be extended to a ring epimorphism $\beta^{\prime}: K[G] \rightarrow K[G / N]$. Since, $G$ is finite and $w K[G]$ is right strongly 2-generated, then $w(K[G / N])$ is right principal and by using ([3] Lemma 4.3) $G / N$ is a $p^{\prime}$-group-by-cyclic $p$-group. If $N$ is a $p^{\prime}$-group, then $G$ itself is a $p^{\prime}$-group-by-cyclic $p$-group. So, we can assume that $|N|=p$, therefore there exists a normal subgroup $F$ of $G$ such that, $F / N$ is a $p^{\prime}$-group and $G / F$ is a cyclic $p$-group. Hence, $(|F / N|,|N|)=1$ and by ([2], Haputstaz 18.1), there exists a complement subgroup $L$ of $N$ in $F$, where $L$ may be taken as the cosets representatives of $N$ in $F$ and $F$ is the semidirect product of $N$ by $L$, it is clear that $L$ is a $p^{\prime}$-group. Let $C=\left\{g \in F \mid g^{-1} n g=n\right.$ for each $\left.n \in N\right\}$, this is a subgroup of $F$ and $N$ is a centeral subgroup of $C$. Hence, $C=N \oplus F^{\prime}$, where $F^{\prime}$ is a subgroup of $L$ and it is not trivial by our assumption on the order of $G$. Since $N$ is a Sylow normal subgroup of $F, N$ is a characteristic subgroup of $F$. So, suppose that $\alpha \in A u t(F)$ and $g \in F^{\prime}$, then $\alpha(n)=\alpha\left(g^{-1} n g\right)=\alpha(g)^{-1} \alpha(n) \alpha(g)$. Hence, $\alpha(g)$ centralizes $N$. Consequently $F^{\prime}$ is a characteristic $p^{\prime}$-subgroup of $F$, hence a normal $p^{\prime}$-subgroup of $G$. As we have shown above $w\left(K\left[G / F^{\prime}\right]\right)$ is a right principal ideal and by ([3] Lemma 4.3$) G / F^{\prime}$ is a $p^{\prime}$-group-by-cyclic $p$-group. Hence, $G$ itself is an extension of a $p^{\prime}$-group-by-cyclic $p$-group.

(ii)-(iii) Is clear by ([3] Lemma 4.3)

(iii)-(i) Is trivial.

Theorem 2. Suppose that $G$ is an infinite solvable group, $K$ is a field of char $p>0$ and that $K[G]$ is a right Noetherian group ring of $G$ over $K$. Then 
$w(K[G])$ is right strongly 2-generated iff $G$ is a finite $p^{\prime}$-group-by-infinite cyclic.

Proof. $(\Rightarrow)$ Suppose that $G$ is a solvable group then it has a normal series $G=G_{0} \triangleright G_{1} \triangleright-\triangleright G_{n}=<1>$, with 1 the identity of $G$, such that $G_{i} / G_{i+1}$ is abelian. Hence, $H=G_{n-1}$ is a non trivial abelian subgroup. We shall prove our theorem by considering the different possibilities of $H$. First suppose that $H$ is a finite group. Assume that $P|| H \mid$, then by the same argument used in propositionl and by ([3] Theorem 4.1), it follows that $G$ itself is a finite $p^{\prime}$ group-by-infinite cyclic. If $p|| H \mid$, then as we have shown above, $G / H$ is a finite $p^{\prime}$-group-by-infinite cyclic, therefore there exists a normal subgroup $F$ of $G$ such that $F / H$ is a finite $p^{\prime}$-group and $G / F$ is infinite cyclic. So, for some element $y \in G$ of infinite order modulo $F, G=\langle F, y>$ and $F=\langle H, L>$, where $L$ is a subgroup of $F$ of the cosets representatives of $H$ in $F$ and it is clear that $L$ is a finite $p^{\prime}$-group. Since, $F$ is a finite normal subgroup of $G$ and $y$ is of infinite order modulo $F$, then there exists an integer $m \geq 1$ such that $y^{m}$ centeralizes both $F$ and $y$. Hence, $\left\langle y^{m p}\right\rangle$ is a normal subgroup of $G$. Consequently $\bar{G}=G /<y^{m p}>=<F, y>/<y^{m p}>$ is a finite homomorphic image of $G$. Since, $w(K[\bar{G}])$ is principal as a right ideal then it is easily shown that $\bar{G}$ is a finite $p^{\prime}$-group-by-cyclic $p$-group. Since, $\bar{y}^{m}$ is of order $p$ which is not contained in $\bar{F}$, we conclude that $\bar{F}$ is a $p^{\prime}$-group. Let $\alpha: F \rightarrow F /<y^{m p}>$ be a group epimorphism, since $y$ is of infinite order modulo $F$, then ker $\alpha=\langle 1\rangle$. Hence, $\alpha$ is a group isomorphism and $F$ is a $p^{\prime}$-group which contradicts that $F=\langle H, L\rangle$ and $P|| H \mid$. This contradiction shows that $H$ must be a $p^{\prime}$ group and as above the result follows. Next, suppose that $H$ is an infinite group and let $T(H)$ be the torsion subgroup of $H$. Since, $H$ is abelian, then $T(H)$ is a finite characteristic subgroup of $H$, hence finite normal subgroup of $G$. If $T(H) \neq<1>$ from the first case, we can easily conclude that $T(H)$ is a finite $p^{\prime}$-group. Consequently $G$ is a finite $p^{\prime}$-group-by-infinite cyclic. So, suppose that $T(H)=<1>$, since, $K[G]$ is Notherian, then $H$ is a finitely generated group. So, let $H=\left\langle x_{1}>\oplus \ldots \oplus<x_{m}\right\rangle$, where $x_{i}, i=1, \ldots, m$ are torsion free elements. Let $H^{p}=\left\{x^{p} \mid x \in H\right\}$, this is a characteristic subgroup 
of $H$, hence normal in $G$ and $H / H^{p} \simeq \bigoplus_{1}^{m} z_{p}$. So, we can deduce that $G / H^{p}$ is a finite $p^{\prime}$-group-by-infinite cyclic. Therefore, there exists a normal subgroup $M$ of $G$, such that $M / H^{p}$ is a finite $p^{\prime}$-group and $G / M$ is infinite cyclic. So, $M=\left\langle H^{p}, L^{\prime}\right\rangle$, where $L^{\prime}$ is a finite $p^{\prime}$-group of the cosets representatives of $H^{p}$ in $M$ and $G=\langle M, u>$ for some element $u \in G$ of infinite order modulo $M$.

Then $\bigoplus_{1}^{m} z_{p} \simeq H / H^{p}=H / H^{p} / H / H^{p} \cap M / H^{p} \simeq H M / H^{p} / M / H^{p} \triangleleft$ $G / M$. Hence $\bigoplus_{1}^{m} Z_{p} \simeq H / H^{p}$ must be cyclic as a subgroup of $G / M$, so we conclude that $H$ is infinite cyclic. Consequently, let $H=\langle x\rangle$ for some torsion free element $x \in G$. Now, by the above procedure we get that $G / H$ is a finite $p^{\prime}$ group-by-infinite cyclic, thus $N / H$ is a finite $p^{\prime}$-group for some normal subgroup $N, G=<N, g>$ for some $g \in G$ of infinite order modulo $N$ and $N=\langle H, B>$, where $B$ is a finite $p^{\prime}$-group of the cosets representatives of $H$ in $N$. Since, $H$ is a infinite cyclic normal subgroup of $G$, then $H^{p}=\left\langle x^{p}\right\rangle$ is a normal subgroup in $G$. Hence, $N / H^{p}$ is a finite normal subgroup of $\left.G / H^{p} \simeq<N, g\right\rangle / H^{p}$ and $g / H^{p}$ is of infinite order-modulo $N / H^{p}$, so there exists $n^{\prime} \geq 1$, such that $g^{n^{\prime}} / H^{p}$ centeralizes both $N / H^{p}$ and $g / H^{p}$. Consequently, $\left\langle g^{n^{\prime}} / H^{p}\right\rangle^{p} \simeq<g^{n^{\prime} p}>/ H^{p}$ is a normal subgroup of $G / H^{p}$. The group $<g^{n^{\prime} p}>/ H^{p} \simeq H^{p}<g^{n^{\prime} p}>/ H^{p}$, since $<g^{n^{t} p}>\cap H^{p}=<1>$ by the choice of $g$ and $H^{p}$ is a normal subgroup of $G$. Therefore, $\left.\left.G / H^{p} / H^{p}<g^{n^{\prime} p}>/ H^{p} \simeq G / H^{p}<g^{n^{\prime} p}\right\rangle=\langle N, g\rangle / H^{p}<g^{n^{\prime} p}\right\rangle$ is a finite homomorphic image of $G$. So, it can be easily shown that $G / H^{p}<$ $g^{n^{\prime} p}>$ is a $p^{\prime}$-group-by-cyclic $p$-group. Since, $g^{n^{\prime}} / H^{p}<g^{n^{\prime} p}>$ is of order $p$ which is not contained in $N / H^{p}<g^{n^{\prime} p}>$, then we conclude that $N / H^{p}<g^{n^{\prime} p}>$ is a $p^{\prime}$-group which contradicts that $x / H^{p}<g^{n^{\prime} p}>\in N / H^{p}<g^{n^{\prime} p}>$ is of order $p$. This contradiction shows that $H$ is not a torsion free group. Therefore, $\Rightarrow$ is proved.

$(\Leftarrow)$ Follows easily by Passman's results ([3], Theorem. 4.1).

Lemma 3. Suppose that $R$ is a commutative integral domain of characteristic Zero, $\pi$ is the set of rational primes which are invertible in $R, G$ is an infinite solvable group and $R[G]$ is a right Noetherian group ring. Then $w(R[G])$ 
is a right strongly 2-generated ideal if and only if $G$ is a finite $\pi$-group-by-cyclic.

Proof. $(\Rightarrow)$ Suppose that $G$ is solvable, then it has a normal series, $G=$ $G_{0} \triangleright G_{1} \triangleright-\triangleright G_{n}=\langle 1\rangle$, with 1 the identity of $G$, such that $G_{i} / G_{i+1}$ is abelian. Let $H=G_{n-1}$, assume that $H$ is a finite $\pi$-group, since $w(R[G / H])$ is a right principal ideal, then using ([1], theorem 1$) G / H$ is a finite $\pi$-group-bycyclic. Since, $H$ itself is a finite $\pi$-group, then $G$ is a finite $\pi$-group-by-cyclic. So, suppose that $H$ is finite but not a $\pi$-group (i.e. for some $q \in \pi^{\prime}, q|| H \mid$, where $\pi^{\prime}$ is the set of rational primes which are not invertible in $R$ ). Since, $q$ is not invertible, $q$ is contained in some maximal ideal $P \triangleleft R$ and $R / P$ is a field of char $q>0$. The epimorphism $\tau=R \rightarrow R / P$ can be extended to $\tau^{\prime}=R[G] \rightarrow R / P[G]$. Since, strongly 2 -generated property is inherited by homomorphic images, then $w(R / P[G])$ is a right strongly 2-generated ideal. Since, $R[G]$ is Noetherian, then $R / P[G] \simeq R[G] / P[G]$ is also a Noetherian group ring of $G$ over the field $R / P$ of $\operatorname{char} q>0$. Then by using the same procedure in theorem 2 we get that $H$ is a $q^{\prime}$-group which contradicts that $q|| H \mid$. Consequently, $H$ is a finite $\pi$-group and as above $G$ is a finite $\pi$-group-by-cyclic. Suppose now that $H$ is an infinite group and as $H$ is abelian, thus the torsion subgroup $T(H)$ is a finite normal subgroup of $G$. Using the same procedure in the above theorem we deduce that $T(H) \neq<1>$ is a finite $\pi$-group. Thus $G$ is a finite $\pi$-group-by-cyclic.

$(\Leftarrow)$ Follows easily from Farkas and Sinder's theorem.

Finally I would like to express my deepest gratitude to Professors J. M. Goursaud and M. H. Fahmy, for their helpful suggestions which have improved the results of this paper.

\section{References}

[1] D. R. Farkas and R. L. Snider, "When is the Augmentation ideal principal?" Arch. Math. 33 (1979), 348-350.

[2] B. Huppert, "Endliche Gruppen I", Springer-Verlag, No. 134, Berlin, 1967.

[3] D. S. Passman, "Observation on group rings", Comm. Alg., 5 (11) (1977), 1119-1162. 